\title{
Surveillance des maladies bactériennes invasives dans l'Arctique nord-américain dans le cadre du projet de Surveillance circumpolaire internationale
}

\author{
Desai $\mathrm{S}^{1^{\star}}$, Li YA ${ }^{1}$, Zulz $\mathrm{T}^{2}$, Bruce $\mathrm{M}^{2}$ \\ ${ }^{1}$ Centre de l'immunisation et des maladies respiratoires infectieuses, Agence de la santé publique du Canada, Ottawa \\ (Ontario) \\ ${ }^{2}$ National Center for Emerging Zoonotic and Infectious Diseases, Centers for Disease Control and Prevention, Atlanta, GA, \\ États-Unis
}

*Correspondance: shalini.desai@phac-aspc.gc.ca

\section{Résumé}

Le présent article résume le contenu de la toute dernière réunion du Groupe de travail sur les maladies bactériennes invasives (MBI) du projet de Surveillance circumpolaire internationale (SCI). Le réseau SCI assure la surveillance des maladies bactériennes invasives au sein de la population et offre un mécanisme qui permet de déterminer l'évolution des taux d'incidence et de la résistance aux antimicrobiens. La réunion s'est déroulée au Canada, à Montréal, les 12 et 13 février 2014. Les données examinées comprenaient les données fournies par les provinces et territoires canadiens participants ainsi que par l'État de l'Alaska. Le présent rapport est fondé sur les enregistrements audio de la réunion ainsi que sur les présentations des participants.

Le Groupe de travail sur les maladies bactériennes invasives du projet $\mathrm{SCl}$ s'intéresse plus particulièrement aux maladies invasives causées par Streptococcus pneumoniae (Sp), Neisseria meningitidis (Nm), Haemophilus influenzae (Hi), le streptocoque du groupe A (SGA) et le streptocoque du groupe B (SGB). Les données recueillies sur les maladies causées par chacun de ces organismes jusqu'en décembre 2012-2013 ont été examinées. Bien que l'incidence de certaines de ces maladies invasives évitables par la vaccination ait diminué, l'émergence de souches de Haemophilus influenzae de sérotype a (Hia) a été observée en Alaska et dans le nord du Canada. Un programme de contrôle de la qualité (CQ) interlaboratoire a été mis sur pied pour contrôler les compétences des laboratoires en matière de sérotypage.

\section{Introduction}

La surveillance des maladies bactériennes invasives fournit des renseignements précieux sur l'incidence, la gravité et les caractéristiques biologiques de ces maladies. Le Groupe de travail sur les maladies bactériennes invasives (MBI) du système de Surveillance circumpolaire internationale $(\mathrm{SCl})$ relève du mandat du Groupe de travail sur le développement durable du Arctic Council international (1). Le système SCI est un projet de collaboration internationale rassemblant de nombreux groupes de travail chargés de mener des enquêtes et des études sur les questions pertinentes à la région circumpolaire-nord (2). Le présent article donne un bref aperçu du système $\mathrm{SCl}$ et de ses efforts de collaboration et rend compte de la réunion la plus récente du Groupe de travail sur les maladies bactériennes invasives qui s'est tenue en février 2014.

\section{Contexte}

Le système de Surveillance circumpolaire internationale (SCI) a été fondé en 1999 en tant qu'effort de collaboration entre le Canada et la région arctique des États-Unis (Alaska) en vue de renforcer la surveillance de la pneumococcie invasive parmi les résidents des régions circumpolaires qui, comparés aux habitants des régions du sud des États-Unis ou du Canada, présentaient une incidence plus élevée de la maladie, associée à une proportion importante d'isolats témoignant d'une résistance aux antimicrobiens (2). Le système a continué d'élargir son action au cours des quinze années suivantes et couvre désormais, en 2015, la surveillance des maladies invasives causées par Streptococcus pneumoniae (Sp), Haemophilus influenzae (Hi), Neisseria meningitidis (Nm), le streptocoque du groupe A (SGA) et le streptocoque du groupe B (SGB) au Canada 
(Territoires, Terre-Neuve-et-Labrador et Nord-du-Québec), aux États-Unis (Alaska), au Groenland, en Islande, en Norvège, en Suède et en Finlande $(3,4,5$ et 6$)$.

Les régions du nord des pays circumpolaires ont de nombreux facteurs en commun, notamment sur le plan des caractéristiques démographiques (faible densité de population), de la géographie (régions étendues à faible densité de population, difficulté d'accès aux soins médicaux pouvant nécessiter le transfert des patients vers des zones plus urbaines), du climat (conditions climatiques extrêmes) et des problèmes sociaux (isolement, différences culturelles). En raison de tous ces facteurs, ces régions sont plus proches les unes des autres que des régions du sud de leur pays respectif. Sur les huit pays de la région circumpolaire-nord, la Russie ne participe pas à la surveillance des maladies bactériennes invasives; elle prend toutefois part aux autres initiatives du système $\mathrm{SCl}$, notamment à la surveillance de la tuberculose (7).

Le réseau $\mathrm{SCl}$ constitue un effort de collaboration unique au sein de la région circumpolaire-nord qui allie à la surveillance de la population, la collecte de données épidémiologiques et de laboratoire. Une fois les données confirmées en laboratoire et les données épidémiologiques (notamment âge, sexe, maladie clinique et facteurs de risque) collectées en collaboration avec des fournisseurs locaux, les cas sont identifiés, d'après des définitions de cas standard, par les autorités de la santé publique des entités administratives concernées. Le système repose sur la surveillance de la population au sein de chaque pays et fournit des données épidémiologiques sur les cas individuels ainsi que des données de laboratoire. Nominalement liées au sein de chaque entité administrative, les données sont ensuite anonymisées et intégrées aux données globales de surveillance du système. Elles sont la propriété de chaque entité administrative et sont compilées au centre du Arctic Investigation Programme du Centers for Disease Control and Prevention (CDC), en Alaska. Ces deux facteurs (surveillance de la population couplée à la collecte de données d'épidémiologie et de laboratoire), font l'unicité de cette collaboration au sein de la région circumpolaire-nord. Le groupe se réunit chaque année soit par cyberconférence (WebEx) soit en personne dans le but de discuter des tendances épidémiologiques actuelles et d'offrir un forum pour aborder les questions afférentes aux maladies bactériennes invasives auxquelles la région circumpolaire-nord est confrontée.

\section{Réunion 2014 du Groupe de travail sur les maladies bactériennes invasives (MBI) du projet de Surveillance circumpolaire internationale (SCI) La réunion 2014 du Groupe de travail sur les maladies bactériennes invasives du projet de Surveillance circumpolaire internationale s'est déroulée au Canada, à Montréal. Les participants représentaient le Canada et les États-Unis. Les membres des autres pays n'ont pas pu participer.}

L'objectif de la réunion annuelle est de passer en revue les données sur les maladies bactériennes invasives recueillies par le projet $\mathrm{SCl}$ afin de mieux comprendre l'épidémiologie des cinq maladies bactériennes invasives sous surveillance. La réunion de février 2014 était axée sur les données recueillies de 1999 à 2012/ 2013. Les données provenant des cinq régions du Canada (Nunavut, Territoires du Nord-Ouest, Yukon, Nord-du-Québec, Nord du Labrador) participant à l'effort de collaboration ont été présentées, ainsi que les données provenant de l'État de l'Alaska.

Les données relevées au Canada de 1999 à 2012 dans le cadre du projet SCI ont été examinées en fonction des variables suivantes : organisme, sérotype, année, âge, région, origine ethnique et manifestation clinique. Comparés aux taux nationaux canadiens, les taux moyens d'incidence des maladies invasives causées par Streptococcus pneumoniae, Haemophilus influenzae, Neisseria meningitidis et SGA relevés dans les régions de surveillance circumpolaire étaient nettement supérieurs. Les taux d'incidence du SGB ont été exclus des comparaisons en raison des différences dans la définition de cas; le projet SCI déclare les cas de SGB de tous les groupes d'âge alors que seules les données du groupe d'âge néonatal sont incluses dans les données nationales. Dans les régions de surveillance circumpolaire, le taux d'incidence des pneumococcies invasives, des infections à Haemophilus influenzae et des infections invasives à SGA (SGAi) était plus élevé chez les résidents Autochtones que chez les non-Autochtones. Concernant la pneumococcie invasive et la méningococcie invasive (MI), les taux d'incidence les plus élevés ont été observés chez les enfants de moins de deux ans. Des différences régionales ont été constatées dans l'incidence globale des pneumococcies invasives et des infections à Haemophilus influenzae. L'infection invasive à SGA était la seule maladie à montrer une tendance à la hausse au cours de la période de surveillance, les groupes les plus touchés étant les enfants âgés de moins de deux ans 
et les personnes de plus de 65 ans. La méningococcie invasive avait le taux de létalité le plus élevé et l'infection à SGB, le taux de létalité le plus faible.

En ce qui concerne l'Alaska, les données examinées couvraient la période allant de 2000 à 2013, une attention particulière ayant été accordée aux données de 2011 à 2013. Les données ont été stratifiées par organisme, par année, par âge, par origine ethnique et par sérotype. Si les cas de méningococcie invasive ont diminué au cours de la période de surveillance, une tendance à la hausse a été observée pour l'infection invasive à SGA et à SGB. Les taux d'incidence de toutes les maladies ciblées étaient beaucoup plus élevés chez la population alaskienne autochtone que chez les non-Autochtones de l'État. L'analyse des cas d'infection à Haemophilus influenzae a révélé la présence majoritaire du sérotype a chez les enfants de moins de cinq ans alors que les souches non typables de la bactérie étaient majoritaires parmi la plupart des cas relevés chez les personnes de 5 ans et plus. La déclaration des cas d'infection à SGB est obligatoire pour tous les groupes d'âge en Alaska.

Le Programme de contrôle de la qualité interlaboratoire du réseau SCI a été établi en 1999 dans le but de contrôler la comparabilité des données soumises selon les différentes méthodes employées par les laboratoires du réseau. Le programme, qui dans un premier temps comparait les résultats de sérotypage et la sensibilité aux antimicrobiens de Streptococcus pneumoniae, a été élargi pour inclure le sérogroupage de Neisseria meningitidis, le sérotypage de Haemophilus influenzae et le typage emm des souches associées aux infections invasives à SGA. Les données de sérotypage des souches de SGB n'étant pas collectées par le réseau SCI, leur inclusion dans le programme de contrôle de la qualité n'est pas nécessaire. Le programme de contrôle de la qualité a démontré l'existence d'un degré de corrélation élevé entre les divers centres $(8,9)$. Ceci renforce la notion que les différences ou les similitudes observées entre les données sont vraisemblablement dues à l'existence de réelles différences entre les données épidémiologiques des divers pays et non pas à des résultats aberrants résultant des méthodes différentes employées par les laboratoires.

\section{Conclusion}

Le réseau de Surveillance circumpolaire internationale est un projet de collaboration internationale unique et efficace qui fournit des données de surveillance sur la population ainsi que des données de laboratoire et des données épidémiologiques sur des maladies ciblées. Ce réseau permet d'obtenir des renseignements précieux sur les maladies bactériennes invasives existantes et émergentes au fil de l'évolution du tableau réel de la morbidité. Selon la dernière réunion en date du Groupe de travail sur les maladies bactériennes invasives, la bactérie Haemophilus influenzae de sérotype a à l'origine d'infections invasives est considérée comme un agent pathogène émergent dans l'Arctique nord-américain. D'autres travaux sont prévus pour caractériser la virulence et la gravité clinique des infections dues à cet organisme.

\section{Remerciements}

Les auteurs remercient tous les pays collaborant à l'action du Réseau de Surveillance circumpolaire internationale ainsi que toutes les personnes ayant participé à la réunion : Christine Cash (Gouvernement du Yukon - Ministère de la Santé et des services sociaux), Kianoush Deghani (Conseil cri de la santé et des services sociaux de la baie James - Service de santé publique), Sylvia Doody Keefe (Labrador Health Centre Région sanitaire Labrador-Grenfell), Heather Hannah (Gouvernement des Territoires du Nord-Ouest), Brigitte Lefebvre (Laboratoire de santé publique du Québec), Angela Mullen (Gouvernement du Nunavut - Ministère de la santé), Nadine Abboud, Annie-Claude Bourgeois (coprésidente SCI TB), Jenne Cunliffe, Walter Demczuk, Irène Martin, Monique St-Laurent et Raymond Tsang de l'Agence de la santé publique du Canada, Karen Rudolph et Prabhu Gounder du Centers for Disease Control and Prevention National Center for Infectious Diseases.

\section{Avertissement}

Les constatations et conclusions exprimées dans cet article n'engagent que leurs auteurs et ne reflètent pas nécessairement la position officielle du Centers for Disease Control and Prevention des États-Unis ni celle de l'Agence de la santé publique du Canada. 


\section{Conflit d'intérêts}

Aucun

\section{Financement}

\section{Aucun}

\section{Références}

(1) The Arctic Council. The Arctic Council Secretariat. http://www.arctic-council.org/index.php/en/.

(2) Parkinson A, Bruce MG, et al. International circumpolar surveillance: A network for the surveillance of infectious diseases in the Arctic. EID. 2008 Jan;14(1):18-24.

(3) Helferty M, Rotondo JL, Martin I, Desai S. The epidemiology of invasive pneumococcal disease in the Canadian North from 1999 to 2010. Int J Circumpolar Health. 2013 Aug;72.

(4) Bruce MG, Deeks S, et al. The International Circumpolar Surveillance System for Population-based Surveillance of Invasive Pneumococcal Disease 1999-2005. EID. 2008 Aug;14(1):25-33.

(5) Bruce MG, Deeks S, et al. Epidemiology of Haemophilus influenzae serotype a in the North American Arctic, 20002005. EID. 2008 Jan;4(1): 48-55.

(6) Bruce MG, Zulz T, et al. The epidemiology of invasive Group A streptococcal infection in the North American Arctic, 2000-2006. Clinical Microbiology and Infection. 2008 May;14(s7):483 P2049.

(7) Zulz T, Parkinson A, et al. International circumpolar surveillance: Prevention and control of infectious diseases: 19992008. IJCH. 2009;4.

(8) Reasonover A, Zulz T, et al. The International Circumpolar Surveillance Interlaboratory Quality Control Program for Streptococcus pneumoniae, 1999 to 2008. J Clin Micro. 2011 Jan;49(1):138-43.

(9) Tsang R, Rudolph K, et al. International Circumpolar Surveillance Interlaboratory Quality Control Program for Serotyping Haemophilus influeanzae and Serogrouping Neisseria meningitidis, 2005 to 2009. J Clin Micro. 2012 Mar;50(3):651-6. 